\title{
A remark on product of Dirichlet $L$-functions
}

\author{
by
}

Kirti Joshi (Tucson, AZ) and C. S. Yogananda (Bangalore)

1. Introduction. While trying to understand the methods and the results of [3], especially in Section 2, we stumbled on an identity (*) below, which looked worth recording since we could not locate it in the literature. We would like to thank Dinesh Thakur and Dipendra Prasad for their comments.

2. The identity. For a positive integer $N \in \mathbb{N}$ set $L_{N}(s)=\zeta(s)$ if $N=1$ and for $N>1$ set

$$
L_{N}(s)=\prod_{\chi(\bmod N)} L(s, \chi)
$$

the product taken over all Dirichlet characters mod $N$. We prove

Theorem. For $\operatorname{Re}(s)>2$, the product $\prod_{N=1}^{\infty} L_{N}(s)$ converges absolutely and we have the identity

$$
\prod_{N=1}^{\infty} L_{N}(s)=\frac{\zeta(s-1)}{\zeta(s)}
$$

We need the following lemma.

Lemma. Fix a prime $p$ and let $S_{p}=\{n \in \mathbb{N} \mid(n, p)=1\}$. For any $n \in S_{p}$ let $f(p, n)$ be the order of $p$ modulo $n$. Then the map $S_{p} \rightarrow \mathbb{N}$ given by $n \mapsto f(p, n)$ is surjective with finite fibres (i.e., the inverse image of any number is a finite set).

Proof. Let $k$ be any natural number. We need an $n$ such that $p^{k} \equiv 1$ $(\bmod n)$ and $k$ is the least positive integer with this property. Clearly $n=$ $\left(p^{k}-1\right) /(p-1)$ has this property. Thus the fibre over any $k \in \mathbb{N}$ is contained in the set of divisors of $p^{k}-1$ and hence is finite.

1991 Mathematics Subject Classification: Primary 11M06; Secondary 11Mxx. 
Pr o of (of the Theorem). For $\operatorname{Re}(s)>1$, we have ([2], Lemma 6, p. 72)

$$
L_{N}(s)=\prod_{p \nmid N}\left(1-p^{-f(p, N) s}\right)^{-\phi(N) / f(p, N)}
$$

where $f(p, N)$ is the order of $p$ in $(\mathbb{Z} / N \mathbb{Z})^{*}$. For brevity, we write $f$ for $f(p, N)$ whenever there is no cause for confusion. The convergence of $\prod_{N=1}^{\infty} L_{N}(s)$ is equivalent to the convergence of the series

$$
\sum_{N \geq 1} \sum_{p \nmid N} \frac{\phi(N)}{f(p, N)} p^{-f(p, N) s} .
$$

Note that for $p \nmid N$ we have $1 \leq \phi(N) / f(p, N) \leq \phi(N) \leq N$. As $p^{f} \equiv 1$ $(\bmod N)$ we see that $p^{f}-1=k N$, for some positive integer $k$. However, several different $k, N$ may give rise to the same integer $p^{f}-1$. In any case the number of different $k, N$ corresponds to the number of divisors of $p^{f}-1$. By [1] (Theorem 315, p. 260), we have $d\left(p^{f}-1\right)=o\left(\left(p^{f}-1\right)^{\varepsilon}\right)=o\left((k N)^{\varepsilon}\right)$ for every positive $\varepsilon$ and $p$ sufficiently large. Consequently, the series (2) is majorised by

$$
C \sum_{k} \frac{1}{k^{s-\varepsilon}} \sum \frac{1}{N^{s-1-\varepsilon}}
$$

for some positive constant $C$ and hence it converges for $\operatorname{Re}(s)>2$.

Thus we can interchange the product over $N$ and over $p$ in (1). Hence if we set

$$
L^{p}(s)=\prod_{N \geq 1, \operatorname{gcd}(p, N)=1}\left(1-p^{-f s}\right)^{-\phi(N) / f(p, N)}
$$

we have $\prod_{N} L_{N}(s)=\prod_{p} L^{p}(s)$.

Now put $U=p^{-s}$ and take the logarithmic derivative of $L^{p}(s)$ with respect to $U$ to get

$$
\frac{d}{d U} \log L^{p}(s)=\sum_{N \geq 1,(p, N)=1} \phi(N) \frac{U^{f-1}}{1-U^{f}} .
$$

By formally writing out geometric series and interchanging order of summations we get

$$
\frac{d}{d U} \log L^{p}(s)=\sum_{N \geq 1,(p, N)=1} \phi(N) \sum_{m \geq 1} U^{f m-1}=\sum_{l \geq 1} a_{l} U^{l-1},
$$

where $a_{l}=\sum_{f(p, N) m=l} \phi(N)$.

Now observe that by the Lemma, for any $l \in \mathbb{N}$ and a representation $l=f(p, n) m$ for some $n \in S_{p}, m \in \mathbb{N}$ (in the notations of the Lemma), we have a divisor of $p^{l}-1$. Conversely, any divisor $d$ of $p^{l}-1$ gives a 
representation $l=f(p, d) m^{\prime}$ for some $m^{\prime}$. Therefore

$$
a_{l}=\sum_{f(p, N) m=l} \phi(N)=\sum_{d \mid p^{l}-1} \phi(d)=p^{l}-1 .
$$

Thus we have

$$
\frac{d}{d U} \log L^{p}(s)=\sum_{l \geq 1}\left(p^{l}-1\right) U^{l-1} .
$$

Then by integrating we see that

$$
L^{p}(s)=\frac{1-p^{-s}}{1-p^{-(s-1)}}
$$

which proves the Theorem.

\section{References}

[1] G. H. Hardy and E. M. Wright, An Introduction to the Theory of Numbers, 4th ed., Oxford Univ. Press, 1968.

[2] J.-P. Serre, A Course in Arithmetic, Springer International Student Edition, Narosa Publ. House, New Delhi, 1979.

[3] Y. Taniyama, L-functions of number fields and zeta functions of abelian varieties, J. Math. Soc. Japan 9 (1957), 330-366.

Mathematics Department

University of Arizona

617 N Santa Rita

P.O. Box 210089

Tucson, AZ 85721, U.S.A.

E-mail: kirti@math.arizona.edu
Indian Institute of Science

Bangalore, India E-mail: yoga@math.iisc.ernet.in

Received on 3.6.1998

and in revised form on 16.3.1999 